\title{
CONTROL OF A ROBOTIC ARM FOR TRANSPORTING OBJECTS BASED ON NEURO-FUZZY LEARNING VISUAL INFORMATION
}

\author{
J. Hernández Vicén, Juan Miguel García, Santiago Martínez, C. Balaguer
}

\begin{abstract}
New applications related to robotic manipulation or transportation tasks, with or without physical grasping are being developed. To perform these activities different kind of perceptions are need. One of the key perceptions in robotics is vision. However, camera-based systems have inherent errors which affect the quality of the information obtained. Image distortion slows down information processing and defers data availability to last processing stages, decreasing performance. In this paper, a new approach to correct diverse sources of visual distortions on images in early stages of the data processing is proposed.

The goal of the proposed system/algorithm is the computation of the tilt angle of an object transported by a robot. After capturing the image, the computing system extracts the angle using a Fuzzy filter that corrects all distortions at only one processing step. This filter has been developed by means of Neuro-Fuzzy learning techniques, using data obtained from real experiments. In this way, computing time can be decreased and the performance of the robotic application can be increased. The resulting algorithm has been tried out experimentally in robot transportation tasks in the humanoid robot TEO (Task Environment Operator).
\end{abstract}

Key words: Humanoid, Robots, Non-grasping, manipulation, ANFIS, NeuroFuzzy, Filter.

\section{INTRODUCTION}

Advances in technology are encouraging the development of new applications and new tasks to be accomplished by humanoid robots. Manipulation tasks can be classified in two main groups: Grasping tasks, in which the way to grab an object by the hand of a humanoid robot is studied [1] and Non-Grasping tasks. In this second type of manipulation tasks, the manipulated object is not fixed to the robot through a solid union. That is, the object can be moved by another [2] or can be on an object that the robot is grasping (For example, a bottle on a tray shown in Fig. 1).
Consequently, it is not possible to ensure a proper transportation task. Therefore, the object features must be accurately obtained in order to be capable of reaching a stability control in the future.

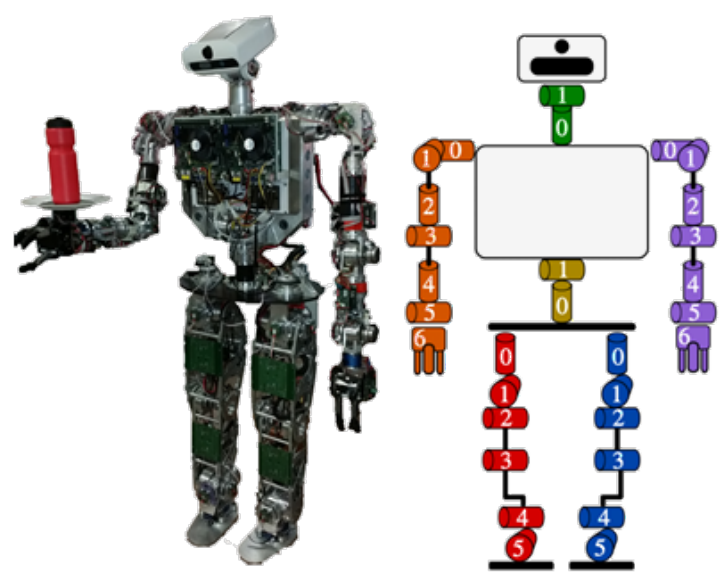

Figure 1: Humanoid Robot Teo Waiter

To obtain those object features, computer vision techniques have been applied. From a camera, the visual information has been transformed into the data needed to control the object. Unfortunately, this visual information is distorted by the inherent error of the camera and by the external errors introduced in the pictures. The method developed has been applied on the example described previously (The bottle on a tray). The information of the bottle obtained by artificial vision has these inherited errors which are produced by the camera and by the perspective between the desired object and the camera. These errors must be corrected to achieve an upcoming control to keep the stability of the transported bottle implemented in the robot, TEO [3].

Therefore, this investigation presents a new method to eliminate the distortion errors related to computer vision. This method lets us to obtain real movement values in an on-line way and without having to rectify the captured images. So, the computation time can be decreased and the performance of the robotic application can be increased. 
The paper is organized as follows: The following section presents how it is obtained the different features of the bottle through the computer vision methods. Section three presents the problems related to the use of computer vision and the new approach proposed to solve it. At the same time, it is shown the ANFIS tool applied to develop the proposed NeuroFuzzy ?lter. In the fourth section, the experiments and results which demonstrate the correction of the error in the system proposed in this research are shown. Finally, some conclusion and prospects for the future are proposed.

\section{VISION FEATURES ACQUISITION}

To accomplish the bottle balance by manipulation techniques following the LIPM, a wide range of features have to be obtained (COG, inclination angle, speed, acceleration). These characteristics to close the control loop have been acquired mainly by computer vision.

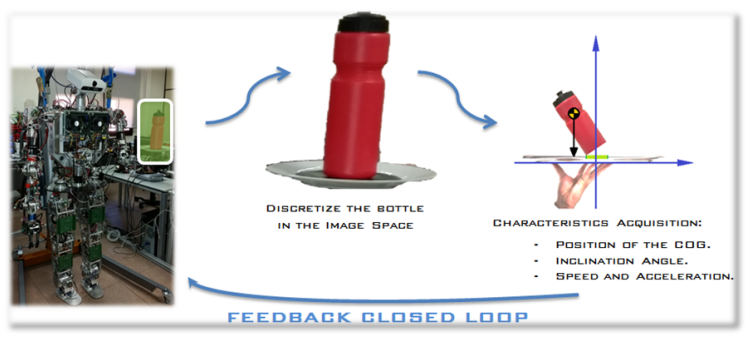

Figure 2: Steps followed to acquire the bottle characteristics and close the loop.

To control the bottle equilibrium, first it is needed to localize it in the space of the image, knowing the situation in which it is in each moment. The inclination angle will be also obtained, to detect if it is in an equilibrium position or otherwise if the humanoid robot must make a correction in the tray to avoid the bottle falling.

Once the tilt angle has been acquired, the angular speed and the angular acceleration will be calculated. These properties will be the inputs to control, in a future work, the robot arm position, closing the feedback loop in order to achieve the bottle balance.

To localize the bottle, the image is discretized separating in the 3 RGB channels, subtracting red and blue channels, and applying a threshold and a filter. After having differenced the bottle from the other objects in the image, the geometrical center has been chosen based on a good reason: As it is geometrically symmetric, its center of mass is the same as its geometrical center.
It is important taking into account that by computer vision we are not able to know the content inside the bottle, so we consider it is filled up with water having constant density. Therefore, we have an advantage in knowing each time the position in the image corresponding with both, the COM and the geometrical center of the bottle.

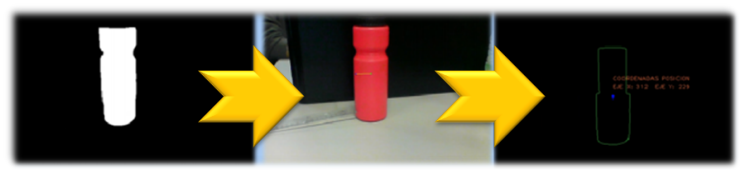

Figure 3: Centre Of Geometry (COG) obtained by computer vision.

\section{NEUROFUZZY LEARNING}

Starting from controlling the balance of the bottle, part of this research has focused on obtaining the angle of the bottle. Through OpenCV tools, the images have been filtered to distinguish the bottle from the rest of the image. And once detected, a box has been attached. The color of the box depends on the inclination value. In other words, the angle of the bottle. Green, if it is stable; orange, if it is losing stability; and finally red, if it is already unstable.

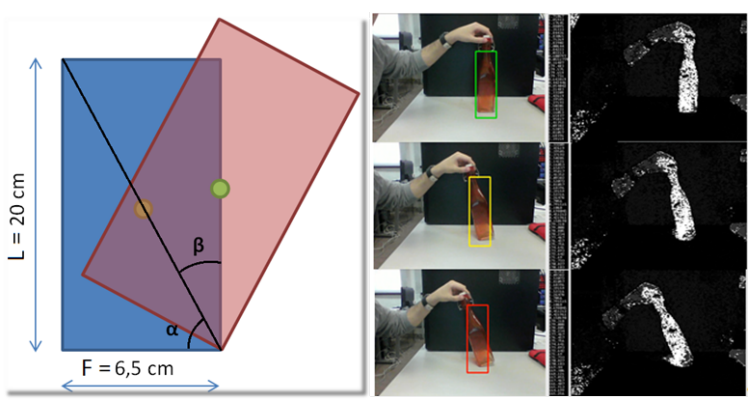

Figure 4: Tilt angle detected by computer vision techniques

However, this visual information is distorted by the inherent error of the camera and by the external errors introduced in the pictures. Errors produced by the camera defects, which are directly related to the camera lenses and another ones caused by the perspective in which the object is being seen.

As the defects introduced by the camera in our system are so low, they have been disregarded. However, high errors are brought in by the perspective in which the bottle is being seen by the camera at each moment. In Fig.5 two images can be seen. In both of them the tray and the bottle are maintained in the same pose. 
The only variation has been made in the orientation of TEO's head. As it can be seen in Fig.5, the image changes completely. In the image (a), it is found the bottle fully straight, and in the other image (b), it is shown the bottle with an inclination. If the real tilt angle is aimed to be obtained directly from these images without using any filter, the data acquired in the first one would be similar to the real angle, whereas the second one would be interpreted wrongly, obtaining an erroneous inclination angle.
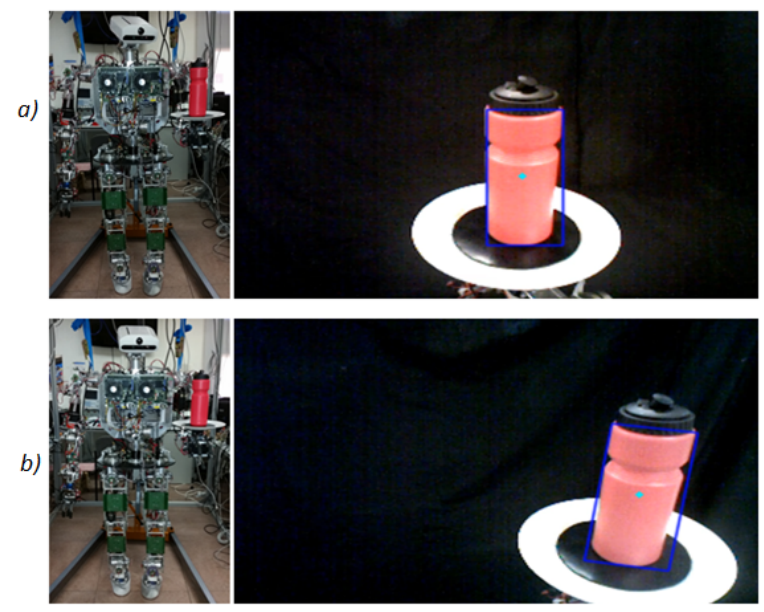

Figure 5: Image showing the perspective error depending of the position of the bottle

A wide range of procedures are available to correct the perspective error [4], also by comparing images taken with different lenses [5] and without calibrating the camera [6]. However, this techniques used to modify the perspective distortion are just based on the correction of the object in the image, in such a way that not mattering the real inclination or orientation, it finally appears as straight as it is explained in Fig. 6 "Classical approaches". These corrections imply an important computational cost. [7].

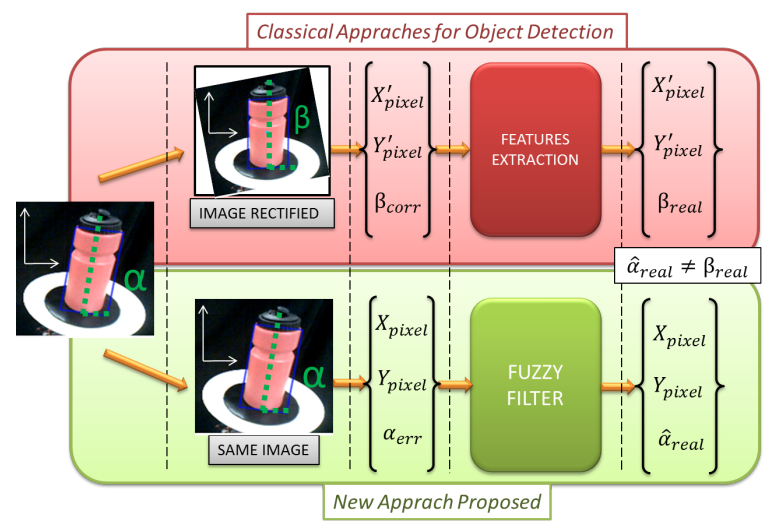

Figure 6: Comparison between classical approaches and the proposed approach in this paper.
Therefore, instead of making a correction on the image and a trigonometric calculus, which takes too much time, an alternative to these classical methods is proposed in this paper, avoiding the complex image manipulation steps needed in the other procedures.

To obtain the correct value of the angle, it is applied a filter based on NeuroFuzzy Learning. the tool used to teach to the filter is called ANFIS (Adaptive Neuro-Fuzzy Inference System). This tool is a multilayer feedforward network in which each node performs a particular function on incoming signals as well as a set of parameters belonging to this node. The formulas for the node functions may vary from node to node, and the choice of each node function depends on the overall input-output function which the adaptive network is required to perform [11][12].

To train the ANFIS system both a training and checking dataset with the information needed have been recorded. In the first one, the performance of the error among the image space introduced in the inclination angle has been recovered by several samplings. These samples have been obtained by positioning the bottle in different controlled positions and inclinations. This information, is used to build the surface to model this error, in order to correct it later. Whereas in the second dataset, information which has been chosen from different positions of the space of the image has been added to have knowledge of all the vision field. This dataset, in which the real inclination angles are well known, are being used to prove that the surface obtained by the ANFIS is reliable.

To validate the information previously acquired by computer vision, the bottle was placed in known and defined positions of the image with known inclination angles. The real data was compared with the calculated data and both were similar. Having reliable information, a training dataset and a checking dataset have been created. Those datasets are needed for the NeuroFuzzy learning based system.

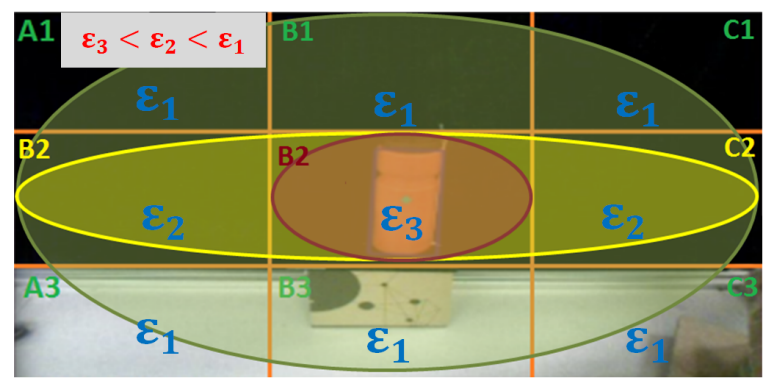

Figure 7: Selection of the Error Priority in the different quadrants of the Image. 
The first one provides the information of the bottle corresponding to the different quadrants of the image, whereas the checking dataset provides the NeuroFuzzy reliable information about the system behavior. As the future goal of this research is to achieve a robust human inspired control [10] of the equilibrium of a bottle on a tray, the image has been divided in nine different zones, according to the accuracy in the correction required for each one of them. This zones are shown in Fig. 7.

The quadrant division has been defined considering the main positions that the bottle occupies in the image. As the head of the robot has been programmed in such a way that it moves trying to keep the bottle centred in the divisions A2, B2 and $\mathrm{C} 2$ seen in Fig. 7, the bottle is positioned most of the time in the three horizontal central quadrants. As those quadrants are occupied by the bottle most of the time, the accuracy in the error correction is higher inside them. As the central quadrant has the highest rate of cases in which the bottle is positioned, the error there must be the lowest in the image.

All this information has been introduced in the Adaptive Neuro-Fuzzy Inference System (ANFIS), available in Matlab. With the ANFIS, the NeuroFuzzy Filter was obtained. The filter is used to make the corrections in the inclination angle of the bottle perceived by the camera. With this correction, the real tilt angle information needed to make the proper rectification of the tray position is finally achieved.

\section{EXPERIMENTS}

In order to prove that the information modified by the NeuroFuzzy filter applied is reliable an experiment has been developed. The bottle has been placed over the tray and the robot has moved the arm in such a way that the bottle stays in the four different quadrants in which the image has been divided.

This experiment has been done positioning the bottle in different angle inclinations. The goal of this experiment is trying if the Fuzzy filter is capable of rectify properly the data obtained by the image, no mattering the inclination angle of the bottle.

As it can be seen in Fig. 8 the robot arm has been commanded in the space of the image. All the movements of the bottle have been recorded by the robot camera. From these movements, the information related to the position in the pixel $\mathrm{X}$ and the pixel $\mathrm{Y}$, as well as the inclination angle acquired by the camera, were obtained and recorded in a table. a)

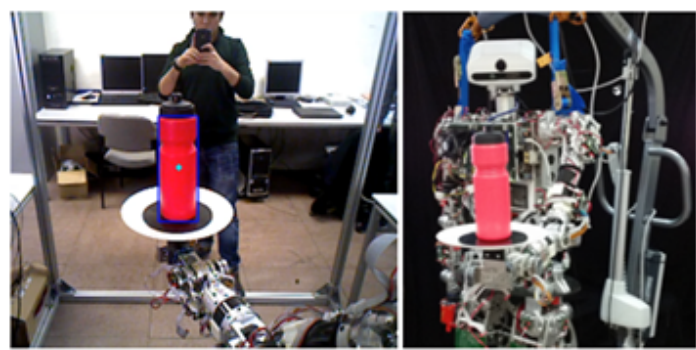

b)

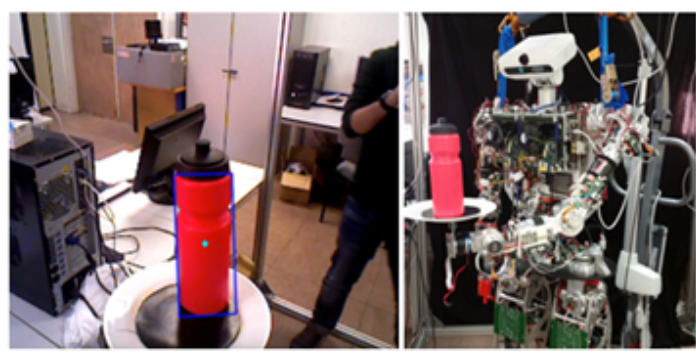

c)

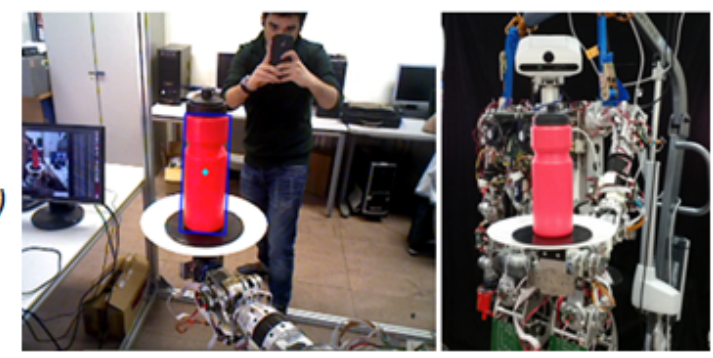

Figure 8: Experiment 01: Positioning the bottle in different inclination angles and positions.

It is important to notice that this tilt angle is not the real one, as far as the image nature is being altered by the errors introduced by the camera and the perspective.

\begin{tabular}{|c|c|c|c|c|c|c|} 
PIXEL X & PIXEL Y & $\begin{array}{c}\text { REAL } \\
\text { ANGLE }\end{array}$ & $\begin{array}{c}\text { ANGLE } \\
\text { DETECTED }\end{array}$ & $\begin{array}{c}\text { FUZZY } \\
\text { CORRECTION }\end{array}$ & $\begin{array}{c}\text { FINAL } \\
\text { ANGLE }\end{array}$ & $\begin{array}{c}\text { ERROR } \\
\%\end{array}$ \\
\hline 270 & 157 & 81 & 68,159 & $-14,9659$ & 83,1249 & 2,62 \\
\hline 26 & 62 & 81 & 93,0146 & $-12,0505$ & 105,0651 & 29,71 \\
\hline 294 & 149 & 99 & 83,405 & $-53,2652$ & 136,6702 & 38,05 \\
\hline 44 & 66 & 99 & 170,354 & $-36,9611$ & 144,3151 & 45,77 \\
\hline 282 & 153 & 87 & 74,0545 & $-14,9475$ & 89,002 & 2,30 \\
\hline 43 & 69 & 87 & 98,7461 & 12,892 & 85,8541 & 1,32 \\
\hline 286 & 148 & 93 & 76,7594 & $-22,6733$ & 99,4327 & 6,92 \\
\hline 35 & 62 & 93 & 103,3924 & 9,8508 & 93,5416 & 0,58 \\
\hline 131 & 67 & 85 & 91,1691 & 5,3272 & 85,8419 & 0,99 \\
\hline
\end{tabular}

Figure 9: Data obtained after applying the NeuroFuzzy filter.

In Fig.9, the inclination angles obtained in the different positions are shown. After applying the NeuroFuzzy filter, this information is corrected and the final angle calculated is presented in the table with the error percentage.

A second experiment was made, in which the try of the robot is moved by the robot in accordance to the information obtained by computer vision and corrected by the NeuroFuzzy filter. In this second case, the robot tries to maintain the bottle in equilibrium over the bottle. 
Inclination Angle of the Bottle

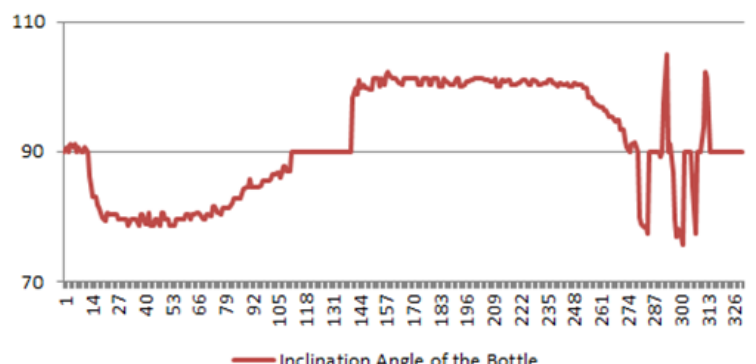

Arm Movement

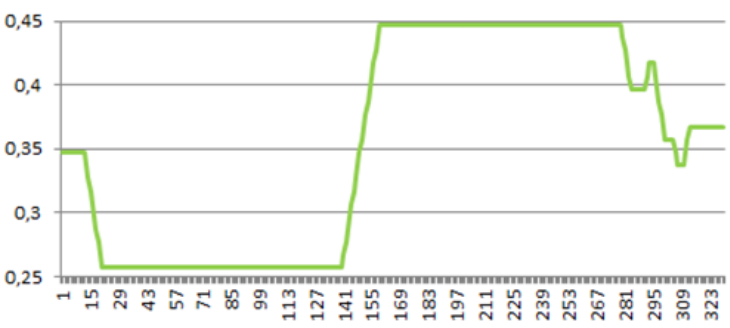

Arm Movement

Figure 10: Corrections made by the arm chain to maintain the bottle equilibrium over the tray depending on the inclination angle.

As it is seen in Fig. 10, when the bottle tilts to one side the robot acts moving the arm in this direction, trying to recover the bottle equilibrium until it reaches the maximum length reachable by the arm.

Whereas, when the inclination angle of the bottle is in the other side, the arm moves to this other side in order to compensate this inclination and keep the balance of the bottle.

\section{CONCLUSIONS}

The conclusions obtained in the research developed and explained in this paper are three. They are exposed below:

- The characteristics of the bottle have been extracted by computer vision: Pre-processing of the image, the inclination angle of the object in each moment, and its movement characteristics.

- The error introduced in the data by the computer vision techniques has been corrected by using a NeuroFuzzy filter.

- Closing the control loop. With the information acquired and corrected, a closed loop has been made. To achieve it, the programs have been implemented in the humanoid robot TEO.

\section{Acknowledgements}

The research leading to these results has received funding from the ROBOHEALTH-A project (DPI2013-47944-C4-1-R) funded by Spanish Ministry of Economy and Competitiveness and from the RoboCity2030-III-CM project (S2013/MIT2748), funded by Programas de Actividades I+D en la Comunidad de Madrid and cofunded by Structural Funds of the EU.

\section{References}

[1] Chua, J. L., Chang, Y. C., Jaward, M. H., Parkkinen, J., Wong, K. S. (2014, December). Vision-based hand grasping posture recognition in drinking activity. In Intelligent Signal Processing and Communication Systems (ISPACS), 2014 International Symposium on (pp. 185-190). IEEE.

[2] Ohno, K., Kensuke, K., Takeuchi, E., Zhong, L., Tsubota, M., Tadokoro, S. (2011, December). Unknown object modeling on the basis of vision and pushing manipulation. In Robotics and Biomimetics (ROBIO), 2011 IEEE International Conference on (pp. 19421948). IEEE.

[3] Martínez, S., Monje, C. A., Jardón, A., Pierro, P., Balaguer, C., Munoz, D. (2012). Teo: Full-size humanoid robot design powered by a fuel cell system. Cybernetics and Systems, 43(3), 163-180.

[4] Jagannathan, L., Jawahar, C. V. (2005, August). Perspective correction methods for camera based document analysis. In Proc. First Int. Workshop on Camera-based Document Analysis and Recognition (pp. 148-154).

[5] Kumar, S., Micheloni, C., Piciarelli, C., Foresti, G. L. (2010). Stereo rectification of uncalibrated and heterogeneous images. Pattern Recognition Letters, 31(11), 1445-1452.

[6] Wu, H. H. P., Yu, Y. H. (2005). Projective rectification with reduced geometric distortion for stereo vision and stereoscopic video. Journal of Intelligent and Robotic Systems, 42(1), 71-94.

[7] Slembrouck, M., Heyvaert, M., Van Cauwelaert, D., Van Hamme, D., Veelaert, P., Philips, W. (2012, October). Time complexity of traditional vision algorithms on a blockbased image processor (BLIP). In Distributed Smart Cameras (ICDSC), 2012 Sixth International Conference on (pp. 1-6). IEEE. 
[8] Trenaman, A., Barry, D., Vernon, D. (1996). An Analysis of Strategies to Reduce Computational Complexity and Processing Time in Industrial Optical Data Processing and Analysis. Proceedings of OEPE'96.

[9] Kim, Y. H., Ahn, S. C., Kwon, W. H. (2000). Computational complexity of general fuzzy logic control and its simplification for a loop controller. Fuzzy Sets and Systems, 111(2), 215-224.

[10] Martínez de la Casa Díaz, S. (2012). Human inspired humanoid robots control architecture.

[11] Jang, J.-S. R., "ANFIS: Adaptive-Networkbased Fuzzy Inference Systems," IEEE Transactions on Systems, Man, and Cybernetics, Vol. 23, No. 3, pp. 665-685, May 1993.

[12] Gómez Vargas, E., Obregón Neira, N., Socarras Quintero, V. (2010). Application of neurofuzzy ANFIS model vs neural network, to the predictive monthly mean flow problem in the Bogotá river in Villapinzón. Tecnura, 14(27), 18-29.

Deben estar ordenadas por orden alfabético y justificadas con la sangría correspondiente. 